\title{
Role of intravitreal dexamethasone in exogenous fungal endophthalmitis
}

\begin{abstract}
Purpose To determine the effects of intravitreal dexamethasone in patients with exogenous fungal endophthalmitis.

Methods Twenty cases of culture-proven exogenous fungal endophthalmitis following cataract surgery $(11 / 20)$ and trauma $(9 / 20)$ were retrospectively analysed for pre- and postoperative visual acuity, anterior chamber and vitreous inflammation and media clarity. All patients were managed with pars plana vitrectomy with intravitreal amphotericin B and oral ketoconazole with (steroid plus group) or without (steroid minus group) intravitreal dexamethasone. Results were analysed by Fisher's exact test.

Results Following vitrectomy 9 of 20 patients (45\%) achieved a visual acuity better than counting fingers at $3 \mathrm{~m}$. No statistically significant difference was observed in anatomical and visual outcome between the steroid plus and steroid minus groups, though the number of patients with favourable visual outcome was greater in the steroid plus group. Rate of clearance of inflammation was better in the steroid plus group ( $40 \pm 15.5$ vs $55 \pm 8.6$ days). All patients $(6 / 20)$ with pre-operative vision better than counting fingers showed good anatomical and visual outcome in both groups.

Conclusions The results of our retrospective study suggest that steroids may be beneficial in promoting faster clearance of inflammation in fungal endophthalmitis. Sensitivity of the fungi to antifungals, dose and timing of steroid and institution of effective antifungal medication prior to the use of steroids are the essential factors which need to be examined further in a prospective manner.
\end{abstract}

Key words Corticosteroids, Dexamethasone, Fungal endophthalmitis, Intravitreal injection

Corticosteroids are potent anti-inflammatory and immunosuppressive agents and are now widely recommended in the treatment of immune-mediated diseases. The antiinflammatory effect of corticosteroids, in
AJIT B. MAJJI, SUBHADRA JALALI, TARAPRASAD DAS, USHA GOPINATHAN combination with antibiotics, has been an area of interest in infectious disorders. Antiinflammatory effects of corticosteroids are utilised to reduce the tissue damage by bacterial toxins in systemic bacterial infections.

Cases of fungal endophthalmitis have increased in recent years, posing a major challenge in their treatment (48 cases of exogenous and 120 of endogenous endophthalmitis were reported world-wide in the last 20 years compared with only 6 cases before 1962). Visual outcome in fungal endophthalmitis continues to be poor (best results were $42 \%$ recovery of visual acuity better than 20/400), despite developments in vitreous surgery and antifungal treatment. ${ }^{1,2}$ It can be of great help in fungal endophthalmitis if the antiinflammatory effects of steroids can be used effectively to avert tissue damage and preserve vision without causing any detrimental effects. Dexamethasone has been beneficial in the faster clearance of inflammation in the treatment of bacterial endophthalmitis, in combination with intravitreal antibiotics. ${ }^{3}$ Detrimental effects of corticosteroids have been postulated in the past, due to their in vitro properties of impairment of the efficacy of antifungal drugs as well as their interference with the immunogenic response that results in further fungal proliferation. ${ }^{4,5}$ Contrary to this, Coats and Peyman ${ }^{6}$ found no evidence of corticosteroid-induced impaired activity of antifungals or enhanced fungal proliferation in a rabbit model using Candida. Elliott and colleagues ${ }^{7}$ reported beneficial effects of topical and systemic steroids in two cases of endogenous fungal endophthalmitis. These two cases showed faster anatomical and visual recovery. Stransky ${ }^{8}$ reported one case of Candida endophthalmitis successfully treated with intravitreal amphotericin B and dexamethasone, where the patient recovered $20 / 20$ visual acuity post-operatively.

To investigate the effect of intravitreal steroid in culture-proven exogenous fungal endophthalmitis, we retrospectively reviewed 20 consecutive patients treated with vitrectomy and antifungal treatment with and without intravitreal dexamethasone. The data were analysed to test the visual and anatomical 
outcome and the rate of clearance of inflammation with steroid use in fungal endophthalmitis.

\section{Subjects and methods}

We reviewed the surgical and microbiology laboratory records to detect culture-positive cases of fungal endophthalmitis in patients who underwent vitrectomy for endophthalmitis at our referral centre between January 1991 and June 1996. We selected cases that were followed up post-operatively for more than 3 months or to a definite surgical outcome. We excluded all patients with mixed fungal and bacterial infections and culturenegative cases. Endophthalmitis resulting from other surgical procedures such as penetrating keratoplasty and glaucoma surgery were not included in the study (as they were in only one group and very small in numbers). Patients with any pre-existing ocular pathology, unrelated to endophthalmitis or vitrectomy, that could affect visual and anatomical outcome were also excluded from the study.

At the time of presentation at our vitreo-retinal service, we assessed visual acuity, intraocular pressure, degree of anterior chamber and vitreous inflammation and fundus details if possible, and performed B-scan ultrasonography if fundus details were not visible. The duration of symptoms and the time between cataract surgery or trauma and presentation were also noted. All patients had surgical intervention within $24 \mathrm{~h}$ of presentation.

After making standard vitrectomy ports, undiluted vitreous samples were collected in all cases, with a $2 \mathrm{~cm}^{3}$ syringe plugged into the suction tubing of the vitreous cutter. The sample was sent immediately to the microbiology laboratory located in the institute with the needle bent in order to prevent air entry into the syringe. The smears were subjected to microscopic evaluation for bacteria (Gram and Giemsa stains) and fungi (potassium hydroxide $10 \%$ and calcofluor white $0.1 \%$ ). The smear report from the microbiology laboratory was available to the operating surgeon within 20 min of sending the sample. The remaining vitreous specimen was inoculated on media such as blood agar, chocolate agar, brain heart infusion broth, thioglycollate broth and Sabouraud's dextrose agar for recovery of bacteria, fungi and Acanthamoeba. ${ }^{9}$

The intraocular drug therapy was chosen on the basis of the initial smear report. Intravitreal antibacterial antibiotics along with dexamethasone $(400 \mu \mathrm{g}$ in $0.1 \mathrm{ml})$ were given whenever the smear was negative for fungus, did not show any organism or was positive for any bacteria. In cases where the smear was positive for fungus, only amphotericin B $(5 \mu \mathrm{g}$ in $0.1 \mathrm{ml})$ was given intravitreally. Where there was a strong clinical suspicion of fungal endophthalmitis, at the discretion of the operating surgeon (A.B.M., S.J., T.D.) amphotericin B alone or in combination with antibacterial antibiotics without dexamethasone was injected intravitreally even if the smear was negative for fungus. Whenever fungus was detected subsequently on culture from smear- negative cases, intravitreal amphotericin B was injected on the same day, and systemic steroids were withdrawn. All patients received topical antifungal agent and, whenever indicated, antibacterial antibiotics and cycloplegics. All culture-proven cases received oral ketoconazole $200 \mathrm{mg}$ twice daily for 4-8 weeks with periodic monitoring of liver function tests.

All patients were assessed post-operatively for visual acuity, anterior segment and vitreous inflammation, ${ }^{10}$ media clarity and retinal status at 1 week, 2 weeks, 1 month, 3 months and every 3 months thereafter.

We compiled the data and divided the patients into two main groups: a steroid plus group if they received intravitreal dexamethasone as mentioned earlier and a steroid minus group if they did not. We assessed the effects of dexamethasone in terms of visual and anatomical outcome and the rate of clearance of inflammation, in post-surgical and post-traumatic groups separately and in combination. We assessed the surgical outcome in comparison with the pre-operative visual status at the time of presentation, irrespective of the treatment groups.

In our study we defined favourable visual outcome as any visual acuity better than finger counting at $3 \mathrm{~m}$, whereas failure to achieve this level was classified as an unfavourable visual outcome. The visual results were converted into decimal values for assessing the significance between groups. Favourable anatomical outcome was defined as clear media with attached retina at 3 months follow-up, with visibility of third-grade retinal vasculature; unfavourable anatomical outcome was defined as failure to achieve clear media at 3 months follow-up, or signs of phthisis bulbi or chronic hypotony resulting in secondary changes. In addition, rate of clearance of the anterior chamber inflammation was noted and compared between the steroid plus and steroid minus groups.

\section{Statistical analysis}

The results were analysed by Fisher's exact test, where all $p$ values less than 0.05 were considered significant.

\section{Results}

A total of 336 vitreous samples from suspected exogenous endophthalmitis were processed during the study period, of which 209 (62.2\%) were found to be culture-positive for either bacteria or fungi. Among these patients, 20 (9.5\%) were positive for fungi alone. These 20 patients form the basis of this report.

\section{Demography}

Of 20 patients with fungal endophthalmitis, 11 had endophthalmitis following cataract surgery and 9 following trauma. Thirteen patients received steroids and 7 patients did not. Fifteen patients were male and 5 were female. The mean age of the patients was 64.1 years (range 50-79 years) in the post-operative endophthalmitis group and 20.4 years (5-36 years) in the 
Table 1. Summary of visual and anatomical outcome, aetiology and duration of follow-up

\begin{tabular}{|c|c|c|c|c|c|c|}
\hline Patient no. & Event & Fungus isolated & Pre-operative VA & Final VA & Anatomical outcome & Duration of follow-up \\
\hline \multicolumn{7}{|c|}{ Steroid minus group } \\
\hline 1 & $\mathrm{ECCE}+\mathrm{IOL}$ & Aspergillus flavus & $20 / 70$ & $20 / 200$ & Favourable & 6 months \\
\hline 2 & $\mathrm{ECCE}+\mathrm{IOL}$ & Dematiaceous sp. & $\mathrm{PL}+\mathrm{PR}$ acc & PL- & Phthisis bulbi & 3 months \\
\hline 3 & $\mathrm{ECCE}+\mathrm{IOL}$ & Aspergillus flavus & $P L+P R$ acc & PL- & Phthisis bulbi & 3 months \\
\hline 4 & Iron $\mathrm{FB}$ & Dematiaceous sp. & $\mathrm{PL}+\mathrm{PR}$ acc & CF $3 \mathrm{~m}$ & Favourable & 4 months \\
\hline 5 & Wooden FB & Aspergillus flavus & $20 / 200$ & $20 / 30$ & Favourable & 16 months \\
\hline 6 & Wooden FB & Hemicola sp. & $\mathrm{PL}+\mathrm{PR}$ acc & PL- & Phthisis bulbi & 3 months \\
\hline 7 & Wooden FB & Aspergillus fumigatus & $\mathrm{PL}+\mathrm{PR}$ acc & PL- & Total RD & 3 months \\
\hline \multicolumn{7}{|c|}{ Steroid plus group } \\
\hline 1 & ECCE & Aspergillus fumigatus & $\mathrm{PL}+\mathrm{PR}$ inacc & PL- & Total RD & 3 months \\
\hline 2 & $\mathrm{ECCE}+\mathrm{IOL}$ & Aspergillus flavus & $\mathrm{PL}+\mathrm{PR}$ acc & PL- & Evisceration & 2 months \\
\hline 3 & $\mathrm{ECCE}+\mathrm{IOL}$ & Dematiaceous sp. & $20 / 200$ & $20 / 40$ & Favourable & 3 months \\
\hline 4 & $\mathrm{ECCE}+\mathrm{IOL}$ & Aspergillus flavus & $\mathrm{PL}+\mathrm{PR}$ acc & PL- & Evisceration & 2 months \\
\hline 5 & $\mathrm{ECCE}+\mathrm{IOL}$ & Aspergillus niger & $\mathrm{PL}+\mathrm{PR}$ acc & PL- & Phthisis bulbi & 3 months \\
\hline 6 & $\mathrm{ECCE}+\mathrm{IOL}$ & Aspergillus terreus & $\mathrm{PL}+\mathrm{PR}$ acc & PL- & Phthisis bulbi & 3 months \\
\hline 7 & $\mathrm{ECCE}+\mathrm{IOL}$ & Aspergillus flavus & $\mathrm{PL}+\mathrm{PR}$ acc & $20 / 30$ & Favourable & 7 months \\
\hline 8 & $\mathrm{ECCE}+\mathrm{IOL}$ & Aspergillus fumigatus & $\mathrm{PL}+\mathrm{PR}$ acc & PL- & Phthisis bulbi & 3 months \\
\hline 9 & Stone FB & Dematiaceous sp. & $\mathrm{PL}+\mathrm{PR}$ acc & PL- & Phthisis bulbi & 3 months \\
\hline 10 & Wooden FB & Aspergillus flavus & $\mathrm{PL}+\mathrm{PR}$ acc & $20 / 100$ & Favourable & 7 months \\
\hline 11 & Thorn & Aspergillus niger & $20 / 50$ & $20 / 40$ & Favourable & 24 months \\
\hline 12 & Wooden FB & Acremonium sp. & CF $1 \mathrm{~m}$ & $20 / 30$ & Favourable & 12 months \\
\hline 13 & Wooden FB & Dematiaceous sp. & CF $1 \mathrm{~m}$ & $20 / 50$ & Favourable & 6 months \\
\hline
\end{tabular}

VA, visual acuity; ECCE +IOL, extracapsular cataract extraction with intraocular lens implantation; FB, foreign body; PL, perception of light; $P R$, projection of rays; acc, accurate; inacc, inaccurate; $C F$, counting fingers; $R D$, retinal detachment.

post-traumatic endophthalmitis group. The mean duration of symptoms was 17.7 days (3-120 days) in post-operative endophthalmitis and 29 days (1-120 days) in post-traumatic endophthalmitis. Aetiology, fungus isolated, visual and anatomical outcome and duration of follow-up are summarised in Table 1.

\section{Comparison of visual outcome between the steroid plus and steroid minus groups}

First, we examined whether the use of steroids was beneficial with respect to visual outcome for all patients. Thirteen patients received steroids and 7 did not. Six of 13 patients $(46 \%)$ in the steroid plus group showed a favourable visual outcome, compared with 2 of 7 patients $(29 \%)$ in the steroid minus group. Though a greater number of patients in the steroid plus group showed a favourable visual outcome, this was not statistically significant $(p=0.64)$.

Next we evaluated the visual outcome with steroid use in post-cataract surgery patients. The steroid plus group showed significant visual improvement over the pre-operative level (25\%), while no improvement was observed in the steroid minus group (0); however, the difference was not statistically significant between the groups $(p=1.0)$. Similarly, we assessed the results in post-traumatic patients. Both the steroid plus and minus groups showed significant improvement over the preoperative level ( $66 \%$ vs $50 \%$ ); however, there was no statistical difference in visual outcome between the groups $(p=0.52)$ (Table 2$)$.

\section{Comparison of anatomical outcome between the steroid plus and steroid minus groups}

Anatomical outcome was analysed to see whether there were any favourable/adverse effects of steroids on the structural integrity of the eye. Thirteen patients received steroids and 7 patients did not. There was no significant difference in favourable anatomical outcome between the two groups $(6 / 13$ vs $3 / 7 ; p=1)$. Seven of 13 patients $(54 \%)$ in the steroid plus group showed an unfavourable anatomical outcome. Five of these 7 eyes progressed to phthisis bulbi and 2 eyes required evisceration. The 2 eyes which required evisceration were positive for Aspergillus flavus. A similar percentage of patients in the steroid minus group $(57 \%, 4 / 7)$ showed an unfavourable anatomical outcome. Three of these 4 eyes progressed to phthisis bulbi and 1 eye showed total retinal detachment (Table 1).

Table 2. Comparison of visual and anatomical outcomes between steroid plus and steroid minus groups

\begin{tabular}{|c|c|c|c|c|}
\hline \multirow[b]{2}{*}{ Outcome } & \multicolumn{2}{|c|}{ Post-surgical } & \multicolumn{2}{|c|}{ Post-traumatic } \\
\hline & Steroid plus & Steroid minus & Steroid plus & Steroid minus \\
\hline \multicolumn{5}{|l|}{ Visual } \\
\hline Favourable & $25 \%(2 / 8)$ & $(0 / 3)$ & $80 \%(4 / 5)$ & $50 \%(2 / 4)$ \\
\hline Unfavourable & $75 \%(6 / 8)$ & $100 \% \quad(3 / 3)$ & $20 \%(1 / 5)$ & $50 \%(2 / 4)$ \\
\hline \multicolumn{5}{|l|}{ Anatomical } \\
\hline Favourable & $25 \%(2 / 8)$ & $33.3 \%(1 / 3)$ & $80 \%(4 / 5)$ & $50 \%(2 / 4)$ \\
\hline Unfavourable & $75 \%(6 / 8)$ & $66.6 \%(2 / 3)$ & $20 \%(1 / 5)$ & $50 \%(2 / 4)$ \\
\hline
\end{tabular}



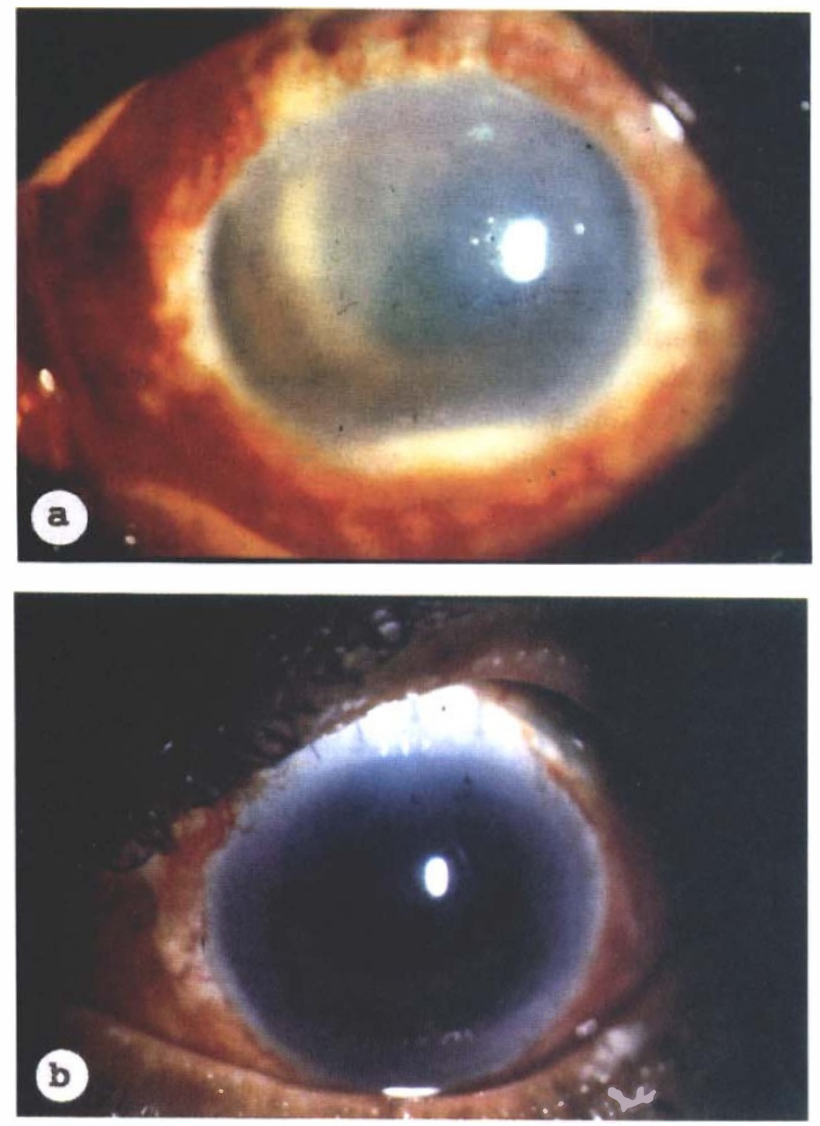

We then tested the anatomical outcome with steroid use in both the post-surgical and post-trauma groups. There was no statistically significant difference in anatomical outcome in either group with steroid use, though more patients in the trauma group showed a favourable anatomical outcome with steroid use $(p=0.52)$ (Table 2).

\section{Rate of clearance of inflammation in steroid plus and} steroid minus groups

We checked whether steroids help in the faster clearance of inflammation. It was found that the rate of clearance of inflammation (flare and cells) in the anterior chamber and vitreous was faster in the steroid plus group compared with the steroid minus group (40 \pm 15.5 days

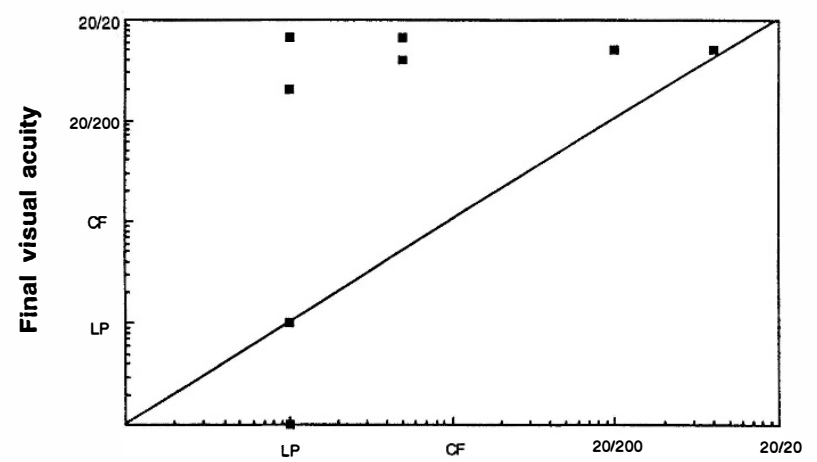

(a)

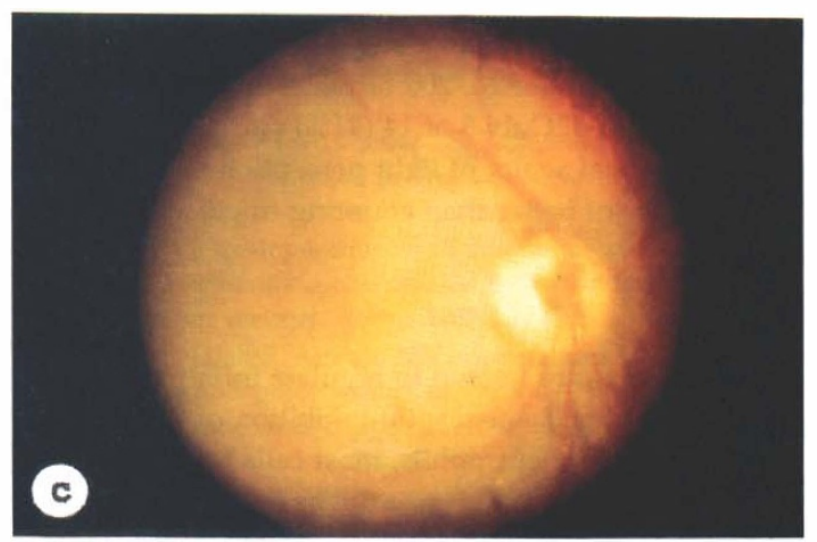

Fig. 1. (a) Pre-operative slit-lamp biomicroscopic picture showing severe anterior chamber inflammation with hypopyon obscuring the fundus details in a patient with Aspergillus flavus endophthalmitis following extracapsular cataract extraction with intraocular lens implantation. (b) Post-operative slit-lamp biomicroscopic picture at 1 month follow-up, showing clearance of the inflammation. (c) Fundus photograph showing clearance of the media and visibility of secondand third-degree vascular branching.

in the steroid plus group compared with $55 \pm 8.6$ days in the steroid minus group) in patients with good anatomical outcome. The clearance of inflammation was good in the steroid plus group irrespective of the fungal growth, as shown in Fig. 1.

\section{Correlation between pre-operative visual acuity and final visual outcome}

We compared the final visual outcome with preoperative visual acuity in both steroid plus and steroid minus groups to assess whether pre-operative visual status had any bearing on the final visual outcome. Favourable final visual outcome correlated well with the pre-operative visual acuity of counting fingers at $1 \mathrm{~m}$ or better (Fig. 2). All patients with pre-operative visual

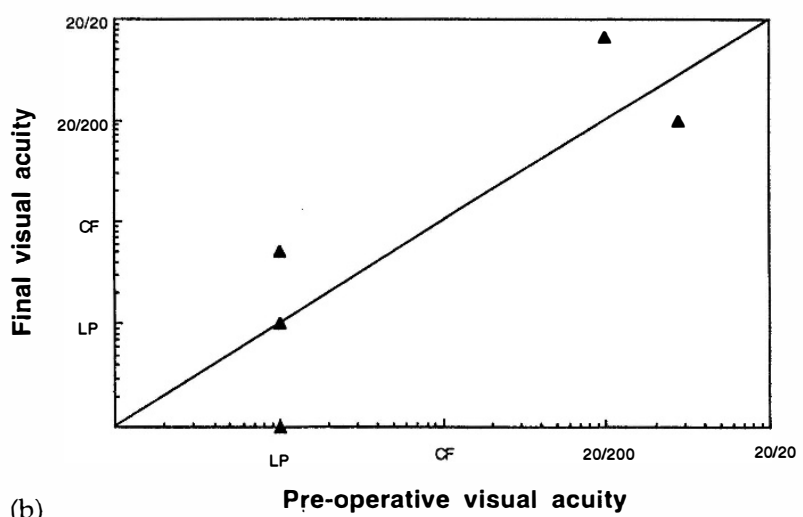

Fig. 2. Comparison between pre-operative visual acuity and final visual recovery in the steroid plus group $(\mathrm{n}=13)(a)$ and steroid minus group $(\mathrm{n}=7)(b)$, showing a positive correlation between pre-operative vision better than counting fingers with a favourable final visual recovery. LP, light perception; $C F$, counting fingers. 
acuity better than counting fingers at $1 \mathrm{~m}$ recovered a final visual acuity of $20 / 200$ or better in the postoperative period. Only 3 of $14(21 \%)$ patients with a preoperative visual acuity of light perception improved to a visual acuity of better than counting fingers at $3 \mathrm{~m}$.

\section{Discussion}

Corticosteroids are known to regulate inflammation by the following mechanisms: (a) inhibition of

degranulation of neutrophils, mast cells and basophils, (b) reduced migration of macrophages to the site of inflammation and (c) interference with the processing of antigens by macrophages. ${ }^{4,5}$ These effects are utilised beneficially in systemic and ocular bacterial infections. Fear of enhancing fungal proliferation either by direct facilitation of fungal proliferation or through the aforementioned process has discouraged steroid use in fungal infections in the past.

Newmark et al. ${ }^{11}$ found that combined therapy with diluted corticosteroids and antifungals controlled fungal proliferation and ocular inflammation in experimentally induced Aspergillus fumigatus keratomycosis in rabbit eyes. Coats and Peyman, ${ }^{6}$ in a rabbit model, studied the effects of dexamethasone in exogenous Candida albicans endophthalmitis and found no evidence of impaired efficacy of antifungal drugs. They further suggested that the addition of corticosteroids to an appropriate antifungal therapy for exogenous fungal endophthalmitis promotes the clearance of destructive inflammation with no apparent enhancement of fungal replication. O'Day et al. ${ }^{12,13}$ investigated the efficacy of prednisolone acetate with or without concomitant antifungal treatment and concluded that adverse effects of corticosteroid administration are inversely related to the in vivo efficacy of antifungal agents. Systemic and topical steroids along with antifungals were used successfully to alleviate inflammation in endogenous fungal endophthalmitis in drug abusers. ${ }^{7,8}$ In this study, though the difference was not statistically significant due to the small patient population, a greater number of patients achieved favourable visual recovery and faster clearance of inflammation with steroid use. These findings suggest a beneficial effect of steroids and, more importantly, open an area for prospective assessment of corticosteroid use in fungal infections.

Grasso et al. ${ }^{5}$ showed dose-dependent suppression of phagocytosis of yeasts by dexamethasone-treated macrophages in vitro. Diamond ${ }^{4}$ has also shown similar effects of steroids on human monocytes in vitro and also observed facilitation of fungal proliferation by dexamethasone. However, no antifungals were used simultaneously in either of these studies to test whether dexamethasone facilitates fungal proliferation even in the presence of effective antifungals. Two patients in this study developed necrotising sclerokeratitis with intravitreal dexamethasone (none without steroid use). Both the patients were positive for Aspergillus flavus on culture. This could be an indication of facilitation of fungal proliferation by dexamethasone, as observed in in vitro studies. ${ }^{4,5}$ Intravitreal amphotericin B was used in both cases. The sensitivity of Aspergillus to this drug may have been poor in these cases (a drug sensitivity test was not done, however), leading to dexamethasonefacilitated fungal proliferation. All patients who received similar doses of steroids did not show any detrimental effects with Aspergillus or other fungi. All these patients received intravitreal dexamethasone prior to identification of the fungus on culture, as the smear was negative for fungi. If steroids facilitate fungal proliferation, then all cases would have worsened, but this did not occur in cases in this study or in the cases reported earlier. ${ }^{2,7,8}$ We hypothesise that sensitivity of the fungi to antifungals, and the dose and timing of steroids, play an important role in the successful outcome of treatment in fungal endophthalmitis. These factors could not be studied in this study because of its retrospective nature. It may prove worthwhile to study these aspects in a prospective manner.

Unlike the classical latency period of weeks to months in fungal endophthalmitis, the majority of patients in this study presented within the first 2 weeks after the surgery or trauma, with a clinical picture mimicking bacterial endophthalmitis as shown in Fig. 1a. This emphasised the need to consider fungal endophthalmitis as a possibility in the immediate post-operative period.

Nine of 20 patients $(45 \%)$ achieved a visual acuity better than counting fingers at $3 \mathrm{~m}$ in our study. Surgical intervention resulted in good visual outcome in all patients with a pre-operative visual acuity better than counting fingers at $1 \mathrm{~m}$. This suggests a benefit of early surgical intervention in fungal endophthalmitis, before the visual acuity has reduced to light perception. If the visual acuity has reduced to light perception then intervention may be too late and may result in poor visual recovery in fungal endophthalmitis, since only $21 \%(3 / 14)$ of patients with light perception regained a visual acuity better than counting fingers at $3 \mathrm{~m}$ postoperatively when compared with $100 \%(6 / 6)$ patients with a visual acuity better than counting fingers. Unlike bacterial endophthalmitis, in fungal endophthalmitis late intervention results in poor visual recovery in the light perception group (80\% visual recovery was reported in patients who presented with light perception in bacterial endophthalmitis ${ }^{14}$ ). Our experience suggests that aggressive surgical removal of the involved vitreous reduces the fungal load and thereby facilitates control of infection by antifungal treatment. Therefore we suggest early and aggressive surgical intervention in fungal endophthalmitis.

\section{Conclusions}

The results of our retrospective study suggest that steroids may be beneficial in promoting clearance of inflammation in fungal endophthalmitis. Surgical intervention in patients with a pre-operative visual acuity of counting fingers or better was associated with good visual results. This suggests a benefit of early surgical intervention in fungal endophthalmitis. 
Sensitivity of the fungi to antifungals, dose and timing of steroids and institution of effective antifungal medication prior to the use of steroids are the essential factors which need to be examined further.

This study is supported by the Hyderabad Eye Research Foundation. The authors thank Dr Devesh Srivastava for comments on the manuscript.

\section{References}

1. Jones DB. Therapy of post-surgical fungal endophthalmitis. Ophthalmology 1978;85:357-73.

2. Pflungfelder SC, Flynn HW Jr, Zwickey TA, et al. Exogenous fungal endophthalmitis. Ophthalmology 1988;95:19-30.

3. Das T, Jalali S, Gothwal VK, Sharma S. Dexamethasone in endophthalmitis treatment trials: results of a pilot study. Invest Ophthalmol Vis Sci 1997;38:S484.

4. Diamond RD. Inhibition of monocyte mediated damage to fungal hyphae by steroid hormones. J Infect Dis 1983;174:160.

5. Grasso RJ, West LA, Gray RC, Lein TW. Inhibition of yeast phagocytosis by dexamethasone in macrophage cultures: reversibility of the effects and enhanced suppression in cultures of stimulated macrophages. Immunopharmacology 1983;4:267-78.
6. Coats ML, Peyman GA. Intravitreal corticosteroids in the treatment of exogenous fungal endophthalmitis. Retina 1992;12:46-51.

7. Elliott JH, O'Day DM, Gutow GS, Podgorski SF, Akrabawi P. Mycotic endophthalmitis in drug abusers. Am J Ophthalmol 1979;88:66-72.

8. Stransky TJ. Postoperative endophthalmitis secondary to Candida parapsilosis: a case treated by vitrectomy and intravitreous therapy. Retina 1981;1:179-85.

9. Sharma S, Jalali S, Adiraju MV, Gopinathan U, Das T. Sensitivity and predictability of vitreous cytology, biopsy and membrane filter culture in endophthalmitis. Retina 1996;16:525-9.

10. Hogan MJ, Kimura SJ, Thygeson P. Signs and symptoms of uveitis. I. Anterior uveitis. Am J Ophthalmol 1959;47:163.

11. Newmark E, Ellison AC, Kaufman HE. Combined Pimaricin and dexamethasone therapy of keratomycosis. Am J Ophthalmol 1971;71:718-22.

12. O'Day DM, Ray WA, Robinson R, Head WS. Efficacy of antifungal agents in the cornea. II. Influence of corticosteroids. Invest Ophthalmol Vis Sci 1984;25:331-5.

13. O'Day DM, Moore TE Jr, Arson SB. Deep fungal corneal abscess: combined corticosteroid therapy. Arch Ophthalmol 1971;86:414-9.

14. Results of endophthalmitis vitrectomy study. A randomised trial of immediate vitrectomy and of intravenous antibiotics for the treatment of postoperative bacterial endophthalmitis. Arch Ophthalmol 1995;113:1479-96. 